\title{
Cinémas
}

Revue d'études cinématographiques

Journal of Film Studies

\section{Georges Sadoul et la Fédération française des ciné-clubs ou Contribution à une histoire des usages non commerciaux du cinéma}

\section{Georges Sadoul and the Fédération française des ciné-clubs, or Contribution to a History of Non-commercial Uses of Cinema}

\section{Valérie Vignaux}

Volume 27, numéro 2-3, printemps 2017

URI : https://id.erudit.org/iderudit/1045373ar

DOI : https://doi.org/10.7202/1045373ar

Aller au sommaire du numéro

Éditeur(s)

Cinémas

ISSN

1181-6945 (imprimé)

1705-6500 (numérique)

Découvrir la revue

Citer cet article

Vignaux, V. (2017). Georges Sadoul et la Fédération française des ciné-clubs ou Contribution à une histoire des usages non commerciaux du cinéma. Cinémas, 27(2-3), 179-194. https://doi.org/10.7202/1045373ar
Résumé de l'article

À partir des archives personnelles de Georges Sadoul, l'auteure de cet article s'interroge sur les raisons de son engagement en 1945 et de son désengagement en 1952 de la Fédération française des ciné-clubs. À l'encontre des récits historiques qui ont fait des ciné-clubs les agents d'une reconnaissance esthétique ou patrimoniale du cinéma, ceux-ci participent d'un mouvement culturel plus général - lancé dans l'entre-deux-guerres par Léon Moussinac qui voit la généralisation d'activités de démocratisation et de décentralisation culturelle, ou autrement dit, d'éducation populaire. Compréhension sociale d'un art qui nuance les discours usuellement formulés sur les activités cinéphiliques de l'après-Seconde Guerre et qui, de surcroît, propose une autre définition de l'éducation populaire elle-même. 


\section{Georges Sadoul et la Fédération française des ciné-clubs ou Contribution à une histoire des usages non commerciaux du cinéma}

\section{Valérie Vignaux}

\section{RÉSUMÉ}

À partir des archives personnelles de Georges Sadoul, l'auteure de cet article s'interroge sur les raisons de son engagement en 1945 et de son désengagement en 1952 de la Fédération française des ciné-clubs. À l'encontre des récits historiques qui ont fait des ciné-clubs les agents d'une reconnaissance esthétique ou patrimoniale du cinéma, ceux-ci participent d'un mouvement culturel plus général - lancé dans l'entre-deux-guerres par Léon Moussinac - qui voit la généralisation d'activités de démocratisation et de décentralisation culturelle, ou autrement dit, d'éducation populaire. Compréhension sociale d'un art qui nuance les discours usuellement formulés sur les activités cinéphiliques de l'après-Seconde Guerre et qui, de surcroît, propose une autre définition de l'éducation populaire elle-même.

L'essor des ciné-clubs au cours des années 1950 a amplement été commenté par les acteurs d'alors ${ }^{1}$ et les exégètes d'aujour$\mathrm{d}^{\prime} h \mathrm{ii}^{2}$, mais nulle étude ne s'est intéressée au rôle joué par Georges Sadoul (fig. 1) alors qu'il fut le secrétaire général de la Fédération française des ciné-clubs de 1945 à 1949, puis de la Fédération internationale des ciné-clubs de 1947 à juillet 1957. Son engagement au sein des deux organisations est avéré puisqu'il rédige nombre de rapports et organise les congrès, alors que dans le même temps, il occupe les fonctions de rédacteur en chef du journal Les Étoiles, publie, d'abord occasionnellement puis hebdomadairement, des critiques sur l'actualité du cinéma dans Les Lettres françaises, et poursuit des recherches pour son Histoire 
UN MOUVEMENT D'UNE IMPORTANCE SANS CESSE CROISSANTE

\section{LE RAYONNEMENT DES CINÉ-CLUBS}

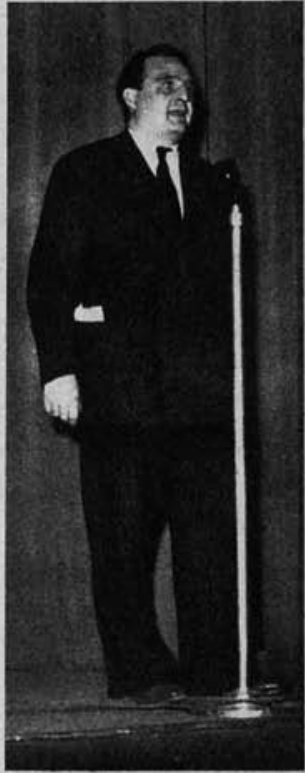

Georges Sadoul, secrótaire général de la Fédération, prononeo son allocution.

A VANT la guerre, le mouve ment des ciné - clubs était encore embryonnaire : quel. ques clubs à Paris, eing ou sts on province. Aucune tentative de sés. Aufourd'hui les gustre-vingt. ses. Aujourd hui les quatre-vingtsix cinc-ciuba roparts sur le terla Fédération groupent 75.000 adhérents. Blentôt, au mois doo tobre, lorsque les auinze ou vingt nouveaux clubs auront été ó liés, le nombre des adhérents đépassera les 100.000 .

Ces chiffres sont une preuvo de In vitailte des cine-clubs ot de leur Federation, dont Jean Painleve vient detre nomme présis'est tenu ces jours-ci a Paris, a mis en rellef l'importance prise dans l'activite intellectuelle du pays par un mouvement qui re pond aux aspirations de tou ceux qui recherchent au ciném. autre chose qu'un divertissemen sans portée.

Les ciné-clubs n'ont pas seulement favorisb 1'6́volution du public en propageant autour d'eux is culture inematographique. In constituent aujourd'hul un do maine experimental dont les créateurs de nims tiennent compte. Grace au public qu'ils ont forme et qui ne cesse de $s^{\prime}$ étendre, un. Inoma a essal pourra blentot nifesteront librement, où de nou- velles formes d'expression pour ront s'élaborer.

Vers une école de moniteurs

Plusieurs résolutions ont été prises au cours du Congrès (auquel assistaient les dirigeants de clubs étrangers). Certaines mért tent qu'on sty arrete :

Fondation d'une foole de moniteurs, oú seront formés les fudirigeanto des cinte-clubs:

Création d'un mowvement destiné à gagner au cinéma de nouveaux eléments du public, encor indifferents, par lorganisation de bibliotheques circulantes, de festivals du film, de présentation exceptionnelles:

Enfn, les congressiates ont voté une resolution demandant letainctubs dun sur formule ne solt utilisée a des fin commerciales ou i la satisfaction commerciaies ou a ia

Dans le rapport qu'il a présenté au Congres, Georges Sadoul, secrotaire general de la Federa. ne bornent pas leur activité aux grands centres, mals étendent maintenant leur action sur de agglomérations de moindre im portance, Bien plus, ils mettent tout en cuvre pour attelndre les publies les plus divers.

\section{Clubs d'entreprises et clubs paysans}

Ainsi, on sait les efforts faite par certains clubs specialisés en faveur du cinema pour lenfance. efrorts dont il faut souhaiter qu'lis aboutissent, en France, a a création d'un cinéma destine la jeunesse. On connalt moins pour lo orétion de clubs popu pires de clubs drentroprises, tel gue celul de fondation rbeonte, des uaines Renault. Ces essats ont té coneluants: ils peuvent etre menés dans maintes directions, par exemple en vue d'atteindre les populations durales par la oreation de cine-cinbs paysans. Georges Sadoul et, après lui, Alexandre Kamenka, devalent insister sur un point particulièrement important: certains explol tants persistent à consideerer les rents Or rlen ne fustife métance pulsque les alms profetés dans les clubs sont prohors d'exploitation. Bien au contraire, en développant chez leurs adnérents le goút du cinéma, les eir.e-clubs contribuent à augmen. te: le nombre des spectateurs des salles a exploitation reguitere. Les exploitants belges lont-11s mieux compris que leurs collègues francals? Un directeur de salle belge, qui assistait à la séance de travall de la salle Marceau, rapportalt qu'a loccasion du Cinquan. Jire dú

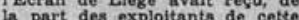
ville, unes exploitants de cette pour cétébrer comme il conventit cet anniversalre.

\section{Une Fédération Internationale}

Enfin, au cours de la sóance đu 28 juin, les délégués français et étrangers déciderent d'un com. mun accord de fonder un comité de liaison international des cinéclubs, qui aurait mission de créer, dans un avenir immédiat, une Feddration internationale des oine-clubs. Ce comite de lialson existe maintenant, et son slego a cte ixe a Parts, il groupe, outre toun de la rance, les represenglque, de littalle, des Pays-Bas te la Pologne. En résumé, oe Congrès aura été une éclatante démonstration de limportance, sans équivalent à 1'étranger, du mouvement des ciné - clubs français. Mouvement dont on ne dirn jamais trop la force qu'il représente pour la dé. fense du cinéma francals ot la culture einématographlque. Jos6 ZENDEL.

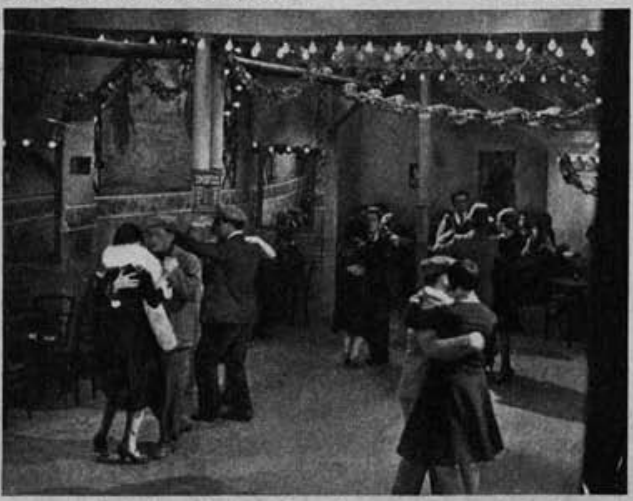

- Sous les toits de Paris $\$$, Mouvre famouse de René Clair, a bt6 présentée, au Palais de Challlot, lors de la étance de eloture du Congros odes Cinb-clubs.

Fig. 1. Photographie de Georges Sadoul au Congrès des ciné-clubs (Zendel 1946, p. 14). 
générale du cinéma dont le premier volume est paru en 1946. Si on peut supposer que ses multiples occupations l'ont incité à se désengager du mouvement, par contre, on peut se demander quelles sont les raisons qui l'ont conduit à collaborer activement à la création de la Fédération. Ainsi, à partir des archives de l'historien et des comptes-rendus parus dans la presse, on s'attachera à retracer les spécificités de cette cinéphilie des lendemains de Seconde Guerre (De Baecque 2003; Jullier et Leveratto 2010); moment singulier concourant à l'écriture d'une histoire des usages non commerciaux du cinéma en France au XX⿳亠丷厂犬⿱

\section{Georges Sadoul}

Lorsqu'est créée la Fédération française des ciné-clubs en mars 1945, Georges Sadoul, qui en est la cheville ouvrière puisqu'en tant que secrétaire général, il signe la plupart des rapports ${ }^{3}$, est âgé d'une quarantaine d'années - il est né en 1904. Il bénéficie au sein de la corporation cinématographique d'une notoriété certaine mais nouvellement acquise, car dans l'entre-deuxguerres, il est plus connu comme spécialiste de l'édition enfantine - il est en effet le rédacteur en chef du journal pour enfant Mon Camarade (Vignaux 2016) ; mais aussi pour ses aspirations littéraires à la suite de son passé surréaliste (Vignaux 2016a) et à la "revue des revues" qu'il réalise pour Commune, l’organe dirigé par Louis Aragon. Sadoul ne devenant spécialiste de cinéma que dans le mitan des années 1930, lorsqu'il s'atèle à la rubrique cinématographique de Regards, ou en raison de ses collaborations à Ciné-Liberté, puisqu'il écrivait le commentaire de L'Espagne vivra (1938), un documentaire militant réalisé par Henri CartierBresson, et présentait des films lors des séances de projection organisées par le collectif (Vignaux 2011). S'il est principalement connu des milieux sympathisants communistes, sa notoriété comme écrivain de cinéma s'est étendue aux lendemains de la Seconde Guerre, car après avoir publié des articles dans L'Écran français clandestin, il est devenu le critique cinématographique des Lettres françaises (Vignaux 2015). Ainsi, lorsqu'il endosse les fonctions de secrétaire général de la Fédération française des cinéclubs, il bénéficie d'une légitimité intellectuelle et morale indéniable, qui contribue à ce qu'il soit une des personnalités 
majeures de ces temps de reconstruction, concourant à nombre des instances nouvellement mises en place et qui vont régir le cinéma des années 1950. Il participe à de nombreuses commissions, appartenant par exemple à la Commission de contrôle des films (censure) au Centre national de la cinématographie, mais aussi à la Commission d'attribution de l'aide à la qualité; il est également membre de la Semaine internationale de la critique à Cannes et siège aussi aux jurys des prix Jean-Vigo et LouisDelluc. Activités auxquelles s'ajoutent ses charges d'enseignement à l'Institut des hautes études cinématographiques et à l'Institut de filmologie, mais aussi ses collaborations aux commissions de recherches historiques mises en place par la Cinémathèque française (Commission de recherches historiques) ou par la Fédération internationale des archives du film (Bureau international de recherches historiques cinématographiques). Autant de responsabilités qui expliqueraient qu'il ait choisi de démissionner en 1957 de ses fonctions de secrétaire général de la Fédération internationale des ciné-clubs. Cependant, si l'on en croit ses archives, il paraît aussi s'éloigner du mouvement. Est-ce à dire qu'il n'en partageait plus les orientations?

\section{La Fédération française des ciné-clubs}

Les rapports ou brochures publiés en ces lendemains de Seconde Guerre et présentant la Fédération comprennent en général un préambule rétrospectif, sans doute rédigé par Sadoul, qui ne manque pas d'affilier le mouvement avec les clubs créés à l'aube des années 1920, témoignant de la sorte d'une saisie historique des événements. Les ciné-clubs de Louis Delluc et Ricciotto Canudo sont systématiquement cités; Léon Moussinac est déclaré être à l'origine de la création du Ciné-club de France puis des Amis de Spartacus; on rappelle que Germaine Dulac présidait dans l'entre-deux-guerres une première confédération; tandis que la Fédération française des ciné-clubs est dite prolonger les visées de Ciné-Liberté. Ces textes participent à la fois d'une volonté didactique et mémorielle, concourant sans doute à une première mythologie du mouvement des ciné-clubs, mais témoignent aussi d'une compréhension des entreprises cinéphiles. Pour les acteurs d'alors, il n'y aurait pas de rupture entre 
l'avant et l'après-Seconde Guerre, continuation assurée grâce à un club particulier, actif à la fin des années 1930, à savoir CinéLiberté. Les animateurs de Ciné-Liberté, sans doute en raison du climat politique du mitan des années 1930, étaient principalement mus par des visées d'éducation populaire ou de démocratisation culturelle: des actions par ou avec le cinéma. Zendel, dans un des premiers textes rétrospectifs sur les ciné-clubs paru en 1947, le souligne également: "[Ciné-Liberté] était le point de départ d'une vaste expérience d'éducation populaire» (p. 13) Cette compréhension du cinéma s'inscrit dans les réflexions sur l'art social, développées au tournant des XIX et $\mathrm{XX}^{e}$ siècles, en raison de l'émergence de nouvelles techniques de reproduction concourant à la structuration des médias de masse. Pensée où les hiérarchies esthétiques érigées à partir des œuvres filmiques sont subordonnées à une émancipation des spectateurs à la suite d'usages didactiques et égalitaristes du cinéma. Ciné-Liberté est, en effet, un club d'un genre nouveau car s'y élaborent conjointement des activités de réalisation de films indépendants, associant amateurs et professionnels, et des séances d'initiation à la culture cinématographique au cours de séances de projection où les films de répertoire ou militants sont commentés. La continuité affirmée entre Ciné-Liberté et la Fédération française des ciné-clubs résulte probablement du fait que la seconde est animée par des personnalités agissantes au sein du premier: Georges Sadoul, déjà cité, mais également Jean Painlevé qui présida les deux groupements, remplacé en 1956 par Jacques Becker, également membre actif du premier club puisqu'il y réalisa La grande espérance (1937), un film documentaire et militant.

La Fédération française des ciné-clubs est créée en mars 1945 à l'initiative de Raymond Bardonnet - ancien membre de CinéLiberté - qui, chargé des questions de distribution et d'exploitation au sein du Comité de libération du cinéma français, une organisation issue de la Résistance, propose à Jean Painlevé, alors à la tête de la Direction générale du cinéma, que soit instaurée une section ciné-club afin de rassembler les six associations déjà constituées. La Fédération regroupe: «Les six aspects d'un mouvement qui s'adresse à la fois aux enfants et aux étudiants, aux techniciens, aux passionnés et aux spectateurs éclairés» 
(Jeander 1949, p. 391). Soit le Ciné-club de l'enfance de Marie Lahy-Hollebecque ${ }^{4}$; le Club Cendrillon de Sonika Bo ; le Cinéclub universitaire; le Cercle technique de l'écran; Ciné-Liberté (manifestement en reprise d'activité) et le Cercle français du cinéma dirigé par Henri Langlois. Dans le troisième Bulletin de l'IDHEC, paru en juillet 1946, on trouve un descriptif assez précis de son fonctionnement:

La Fédération est administrée par un Conseil composé de quinze membres au moins et de trente-cinq au plus, choisis parmi les délégués des ciné-clubs, de la Fédération des spectateurs et de la Cinémathèque [française]. [...] La Fédération fournit à ses adhérents des programmes composés de films provenant de la Cinémathèque [française], de son fonds propre, ou des maisons de distribution et de production. Chaque programme est accompagné de notices et de schémas de conférences ou de discussions. Il existe à l'heure actuelle cinquante programmes environ ${ }^{5}$.

Mais aussi de ses visées :

La connaissance du cinéma: par la fréquentation des classiques impossibles à connaître par voie de distribution normale. [La c]ontribution au développement d'une culture cinématographique: par des débats accompagnant la projection des films, des réflexions, des critiques, tendant à donner aux spectateurs une formation étendue et solide. [L'e]xtension de cette culture: par la conquête de nouveaux spectateurs, en vue d'accroître le rayonnement du cinéma. [La $\mathrm{m}$ ]ise en contact du public et des réalisateurs du film: par des conférences qui resserrent ainsi les liens établis entre les techniciens du film et la masse du public. [La D]éfense du cinéma: par la presse et la propagande, en essayant d'encourager les bons films et de combattre les mauvais ${ }^{6}$.

Les premières séances commentées organisées par la Fédération ont été présentées par Louis Daquin, Jean Grémillon, Jean Painlevé, Georges Sadoul, Claude Aveline, Jacques Becker, Jacques Prévert, Nicolas Hayer ou Jean-Paul Le Chanois ${ }^{7}$. Pour la plupart membres de Ciné-Liberté, ils sont proches du Parti communiste et se sont révélés actifs dans la Résistance, appartenant au Comité de libération du cinéma français. Ils ont accompagné la 
"cinquantaine» de programmes composés de "bons" films et contribuant à "défendre" le cinéma. Or, si l'on en croit la liste (fig. 2) établie par Sadoul pour la saison 1947-1948 et les thématiques de la revue Ciné-Club, l'organe du mouvement, les programmes comprennent quasi exclusivement des films français réa-

\section{Fiches F.F.CC 1947.1948}

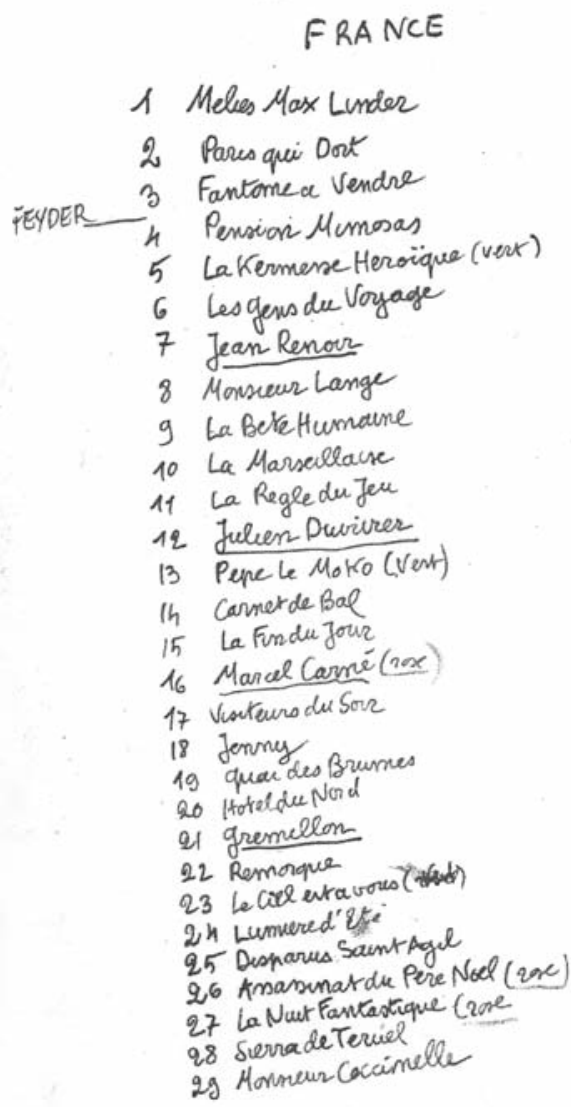

Fig. 2. Inventaire des fiches de la Fédération française des ciné-clubs, établi par Georges Sadoul. Archives privées Georges Sadoul.

Georges Sadoul et la Fédération française des ciné-clubs ou Contribution à une histoire des usages non commerciaux du cinéma 
lisés par des cinéastes proches des premiers groupements (René Clair, Jean Renoir, Jacques Feyder) ou reconnus pour leurs qualités d'artistes (Julien Duvivier, Marcel Carné, Jean Grémillon), auxquels on a associé des films étrangers dont les valeurs esthétiques ont été soulignées par la critique, qu'ils soient "anciens» (films burlesques ou soviétiques) ou "contemporains" (à l'instar de Welles par exemple). Le mouvement des ciné-clubs semble donc prolonger à l'identique une conception littéraire et artistique du cinéma, formulée dès les années 1920, où le cinéaste est envisagé comme metteur en scène et auteur, et le film comme écriture et œuvre. Ainsi, la cinéphilie véhiculée par la Fédération en ces lendemains de Seconde Guerre emprunterait aux ciné-clubs des années 1920 (Gauthier 1999) leurs discours savants et à CinéLiberté ses visées de démocratisation culturelle. Objectifs qui ne semblent pas contradictoires aux promoteurs du mouvement, partagés entre promotion d'un cinéma de "qualité" - Ciné-Club ressemble bien plus aux organes "cinéphiliques", créés par Louis Delluc, qu'au bulletin destiné aux militants et essentiellement pragmatique diffusé par Ciné-Liberté - et volonté d'employer le film à des fins d'émancipation des spectateurs, puisqu'à nouveau la réalisation de films indépendants est encouragée. Les promoteurs du mouvement voudraient qu'à chaque club cinéphilique soit associé un groupement de cinéastes, espérant réitérer ce qui constituait la singularité de Ciné-Liberté:

Création, dans chaque club, d'une section de cinéastes amateurs qui le doublerait. Si cette section existe déjà, essai de collaboration. Enfin, la Fédération qui groupe une centaine de ciné-clubs représentant une force commerciale importante, il semble possible de concourir à la naissance de films d'essai, dont l'amortissement serait rendu parfaitement possible par les ciné-clubs français et étrangers. Ce qui permettrait de donner au cinéma français une vie nouvelle et de larges possibilités de recherches ${ }^{8}$.

Ambivalence qu'on retrouve entérinée dans le compte-rendu du congrès national organisé en juin 1947, lorsque sont définis les objectifs de la Fédération, à savoir la projection de classiques de l'écran en séances commentées, la publication d'un bulletin, et la réalisation de films indépendants ou d'essai. Modèle qui prolonge à l'identique des expériences antérieures, issues des 
années 1920 et 1930, mais qui sera l'objet d'une transformation inattendue en raison d'une donnée nouvelle, propre à ces lendemains de Seconde Guerre, soit la très grande expansion provinciale du mouvement.

\section{La FFCC: une cinéphilie des années 1950}

À sa création en 1945, la Fédération regroupe les six associations existantes. Or, dès 1946, celles-ci sont au nombre de 83, pour atteindre 180 clubs au début des années 1950; chiffre qui commence à décroître à partir de 1952. Groupements qui, au plus fort de leurs actions, comprenaient 100000 membres, nombre apparemment jamais dépassé, avant de se stabiliser dans le mitan des années 1950 à 50000 adhérents. Dans leur ensemble, les clubs n'organisent qu'une séance par semaine, quarante semaines par an, représentant un potentiel de quatre millions de spectateurs alors qu'on en compte à la même période, dans le cadre de l'exploitation classique, plus de quatre cents millions. Ainsi, si le mouvement est conséquent, ce n'est évidemment pas en fonction d'un éventuel succès quantitatif - en dépit des récriminations des exploitants qui font état d'un probable manque à gagner, augmenté d'une concurrence déloyale, car les clubs ne sont pas assujettis à la taxe sur les spectacles — mais bien plutôt en raison d'une large pénétration sur le territoire national d'un catalogue de films de "répertoire», pour des séances comprenant une part importante de prestation orale. Georges Sadoul le constate également lorsqu'il écrit en septembre 1945: «Les ciné-clubs de province, presque inexistants avant 1939, prennent et vont prendre un développement considérable.» Implantation dont on peut suivre l'effectivité dans Ciné-Club, à travers la rubrique "Activités de nos correspondants» (Sadoul 1945), montrant de plus que les clubs ont majoritairement été créés par des membres du corps enseignant, ce que souligne aussi Sadoul lorsqu'il écrit en 1950 :

La Fédération française des ciné-clubs compte 160 clubs et environ 100000 membres. Le recrutement est en majeure partie petit-bourgeois (étudiants, professeurs, instituteurs, etc.) mais comporte dans la plupart des clubs une large proportion de progressistes ${ }^{9}$. 
Animateurs de clubs, qui dans leur très grande majorité, n'ont qu'une faible compréhension de l'art cinématographique et sans doute aucune expérience de réalisation. La Fédération se trouve donc confrontée à deux difficultés: il lui faut répondre à la demande accrue de programmes mais également de connaissances cinématographiques. Ainsi, dès les lendemains de son premier Congrès en septembre 1946, grâce à l'aide matérielle et institutionnelle de la direction des Mouvements de jeunesse et d'éducation populaire, la Fédération organise une session de formation à destination des dirigeants des clubs, regroupant près de 80 personnes pour "des séances de travail sur l'histoire du cinéma dirigées par M. Sadoul, des présentations de films parlants faites par divers critiques, cinq causeries sur divers aspects du cinéma, une séance pédagogique et une visite de studio ${ }^{10}{ }$. Sessions qui seront réitérées chaque année sous forme de stages, dispensés au château de Marly-le-Roi prêté par la direction de l'Éducation populaire du ministère de l'Éducation nationale ou à l'IDHEC, auxquels concourent des professionnels du cinéma. Des techniciens présentent leurs activités, des réalisateurs commentent leurs films, tandis que des critiques traitent de questions historiques - Lotte Eisner interviendra à deux reprises sur le cinéma expressionniste allemand —, ou génériques, comme le western par exemple; interventions qui préludent ou succèdent à des publications, à l'instar de Jean-Louis Rieupeyrout qui, en 1954, fera paraître aux Éditions du Cerf, un opus consacré au Western, préfacé par André Bazin. Les programmes proposés par la Fédération sont systématiquement accompagnés de fiches ${ }^{11}$ destinées à guider la discussion autour du film et, à partir de 1948 , la formation comprend une partie "pratique» où les dirigeants de clubs sont incités à mener eux-mêmes les discussions. Initiation qui se poursuit également au cours de l'année lorsque des professionnels — réalisateurs, acteurs ou techniciens — se déplacent en province pour commenter les films. Les modalités de monstration sont rapidement codifiées: "Dans sa forme actuelle et à quelques très rares exceptions près, [la séance] consiste en une projection d'un ou plusieurs films précédée d'une présentation et suivie d'une discussion générale dirigée par les animateurs du ciné-club»(Jeander 1949, p. 393). Si 
l'initiation à la culture cinématographique paraît avoir trouvé des solutions aisées, il n'en fut pas de même en ce qui concerne l'offre de films :

En septembre 1945, c'est-à-dire au moment où les demandes de création de clubs affluèrent à la Fédération, la programmation devint un problème angoissant et les dirigeants de la Fédération durent courir de distributeurs en distributeurs pour faire la chasse aux chefs d'œuvres [sic] que l'exploitation normale dédaignait (ibid., p. 391-392).

La Cinémathèque française, dans un premier temps, a soutenu le mouvement, mais devant l'expansion numérique des clubs, Henri Langlois s'est rapidement distancié, avant de signifier, par la lenteur des réponses apportées aux courriers, une franche opposition. Dans un texte de 1949, Sadoul mentionne "[une] lutte fratricide entre nos deux organisations pour la domination de l'une sur l'autre ${ }^{12}{ }^{2}$. Défection qui empêche la transmission du cinéma dans son histoire; or, la Fédération, par la diffusion de films anciens, entendait contribuer à une conservation mémorielle du $7^{\mathrm{e}}$ art. Pour pallier cet inconvénient, à la suite d'un congrès qui a réuni les clubs étrangers, Sadoul s'attelle dès septembre 1947 à la création d'une fédération internationale. Il imagine de la sorte pouvoir négocier directement avec la Fédération internationale des archives du film, mais le projet tourne court en raison du mauvais vouloir des dirigeants des cinémathèques étrangères, peu enclins à prêter leurs copies. Un accord est néanmoins entériné en 1950, non sans tracas, mais il peine à être suivi d'effets. Les Archives consentent à prêter leurs films à la Fédération si celle-ci exécute à ses frais des contretypes et des tirages pour mettre à la disposition de ses adhérents des films patrimoniaux (fig. 3). La Fédération, on s'en doute, ne dispose pas des moyens financiers suffisants et il semble que seule La passion de Jeanne d'Arc de Dreyer ait pu être tirée. Difficultés qui expliquent aussi que la majorité des films proposés soit française.

Aux embarras engendrés par la pénurie des programmes, s'ajoutent à partir de 1949, les prétentions de l'administration des contributions indirectes qui souhaite assujettir les clubs à la 


\section{Ciné-屏ub}

2, RUE DE L'ELYYSEE, PARIS - 8' - TELEPHONE ANJOU - 91.54 .55 .56 - C. C. P. PARIS 5397.81

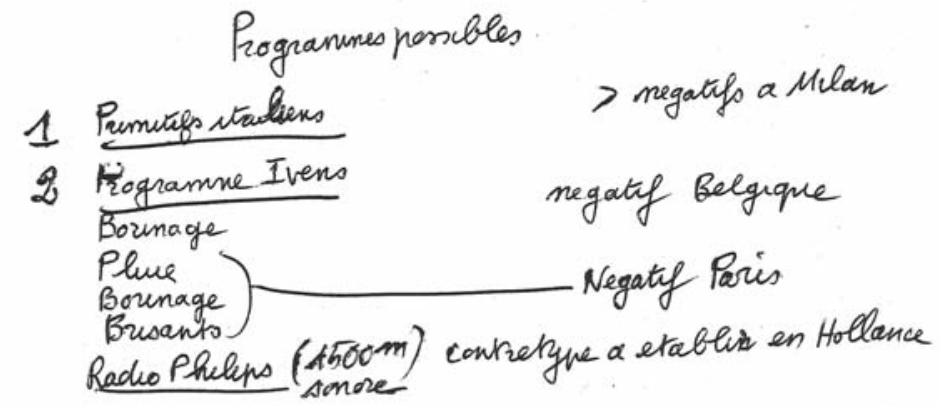

3. Programme

avantgarde fransaus 1920

Souriante Madame Bendet. Negaty Paris

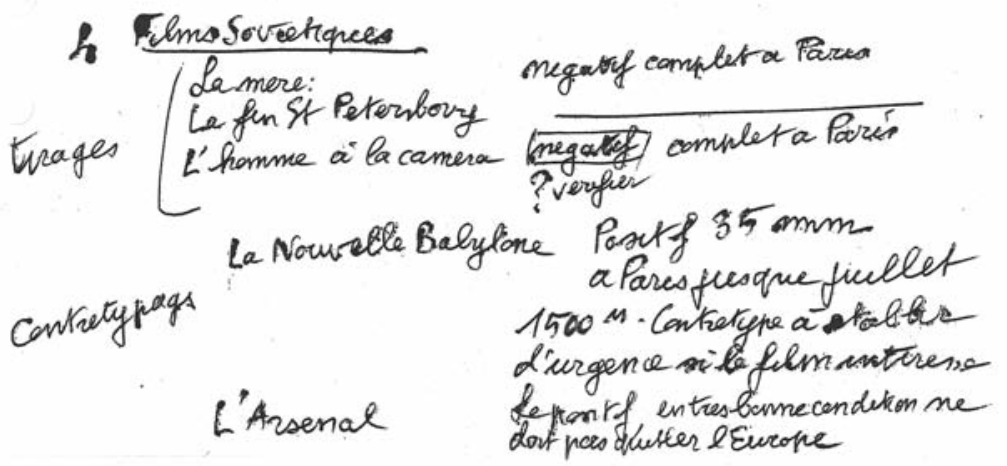

Fig. 3. Programmes de films "possibles» établis par Georges Sadoul, avec indication des cinémathèques conservatrices des éléments. Archives privées Georges Sadoul.

taxe sur les spectacles. L'administration fiscale considère en effet que les séances organisées par les clubs, parce qu'elles montrent du cinéma commercial, ne peuvent être considérées comme des séances culturelles ou éducatives, au sens où celles-ci ont été définies par une circulaire datant de 1929, qui reconnaît 
l'exonération de la taxe sur les spectacles aux «séances à caractère strictement éducatif, comprenant des films scientifiques, agricoles ou documentaires destinés à instruire et non à distraire" (Bloc-Delahaie 1950). Après nombre de procédures (Hamery 2012, p. 75-88), les tribunaux déclareront la circulaire obsolète, et reconnaîtront la valeur éducative des films projetés au cours des séances incriminées. L'attendu publié permet d'imaginer ce que pouvait être une séance de club et la teneur des discussions qui s'y déroulaient :

Attendu que le rapprochement du film Partie de campagne, illustration d'une nouvelle de Maupassant, avec Boudu sauvé des eaux, choisi comme caractéristique d'un montage accéléré des séquences et de l'influence du jeu des acteurs avait pour objet de montrer deux aspects divers de la personnalité d'un metteur en scène, Jean Renoir (Bloc-Delahaie 1950).

Difficultés qui prendront fin avec la parution d'un décret, voté le 21 septembre 1949, réglementant et reconnaissant un possible usage culturel et non lucratif du cinéma commercial. Statut qui couronne les actions de Léon Moussinac, lorsqu'avec obstination, il réclamait pour le cinéma commercial une reconnaissance culturelle. Combat qui l'opposait en 1926, en un procès exemplaire car faisant jurisprudence, au producteur Jean Sapène et qui, en établissant le droit à la libre critique, reconnaissait au film les caractéristiques d'une œuvre de l'esprit. Puis, avec la création en 1928 d'un ciné-club populaire, baptisé les Amis de Spartacus, il montrait, en raison de l'engouement rencontré, de possibles usages éducateurs de l'art cinématographique et réalisait de la sorte une des premières expériences de démocratisation culturelle menée avec le cinéma.

Ainsi, en dépit des oppositions rencontrées, les ciné-clubs, des années 1920 aux années 1950, auront joué un rôle crucial quant à l'histoire du cinéma en France: reconnaissance de qualités éducatives car culturelles du cinéma commercial et de fiction, auparavant concédées aux seuls films didactiques; création de la notion de «répertoire" à travers une liste d'œuvres validée par la critique savante; constitution d'une corporation soudée intéressée à la défense d'un cinéma national ${ }^{13}$; décentralisation 
culturelle et reconnaissance académique (scolaire et universitaire) du cinéma comme moyen ou objet de culture. Aboutissement qui expliquerait le retrait progressif de Sadoul car nombre des contradictions de l'entre-deux-guerres paraissent à présent résolues. Cependant, il semble que la Fédération ait échoué sur l'un au moins de ses objectifs, celui qui préconisait d'employer le cinéma à des fins de démocratisation culturelle, alors qu'il semble qu'au même moment, le théâtre y parvienne, par l'entremise des pratiques amateurs. En effet, les ciné-clubs des années 1950 sont aux mains des classes moyennes qui développent, à partir du cinéma, une culture de classe. Nul essai cinématographique ${ }^{14}$ ne paraît avoir été réalisé dans leur sillage, alors qu'il existe dans le même temps des clubs de cinéastes amateurs, eux aussi réservés aux classes moyennes, en raison des coûts prohibitifs engendrés par la technique. Constat qui pourrait expliquer le désengagement progressif des membres fondateurs, cinéastes et techniciens, issus de Ciné-Liberté, qui confient progressivement la gestion de la Fédération aux seuls animateurs, dirigeants de clubs et membres du corps enseignant. Changement dont témoigne aussi l'évolution de l'organe du mouvement qui, après deux mois sans parution, reparaît en décembre 1949, mais dirigé par Armand J. Cauliez ${ }^{15}$ avant de disparaitre définitivement en 1952. Cinéphilie qu'on peut dire propre aux années 1950, car résultant d'une plus large scolarisation de la société française ainsi que du développement des classes moyennes; évolution qui à l'évidence diverge des visées "progressistes» défendues par Sadoul. Constat qui pourrait l'avoir incité à privilégier ses travaux de critique et d'historien, se retirant de la Fédération française des ciné-clubs en 1949, pour n'y revenir qu'une ultime fois, en 1955, afin de promouvoir Paul Carpita, fondateur de Cinépax, un collectif cinématographique indépendant et réalisateur d'un film militant, $L e$ rendez-vous des quais (1955).

Université de Caen Normandie 


\section{NOTES}

1. Voir Jeander 1949; Chevalier et Egly 1953; Pinel 1964.

2. Voir Loyer 1992; Hamery 2008 ; Souillés-Debats 2017.

3. Fonds Georges Sadoul, Cinémathèque française, GSD5.

4. Marie Lahy-Hollebecque était aux côtés de Léon Moussinac lors de la création du premier ciné-club «populaire», les Amis de Spartacus, en 1928.

5. "La Fédération française des ciné-clubs», Bulletin de l'IDHEC, $\mathrm{n}^{\circ} 3$, juillet 1946, p. 16, je souligne.

6. Ibid., je souligne.

7. Liste établie à partir des comptes-rendus parus dans Ciné-Club, entre 1947 et 1952, année où la revue est remplacée par Cinéma 52.

8. IDHEC, op. cit., je souligne.

9. Lettre de Georges Sadoul à M. Lebediev, chargé de pouvoir de Sovexportfilm, Prague, datée du 7 août 1950, fonds Georges Sadoul, Cinémathèque française, GSD5.

10. IDHEC, op. cit.

11. Les fiches sont établies sur un modèle standard qui comprend: 1. Le générique; 2. Le sujet (a. résumé du scénario; b. la construction dramatique); 3. La réalisation (a. la mise en scène; b. l'interprétation); 4. Importance du film (a. sur le plan artistique; b. sur le plan social) ; 5 . Extraits de presse; 6. Sujets de débats. Cf. fonds Georges Sadoul, Cinémathèque française, GS123, fiches de la Fédération française des ciné-clubs, 1947-1948.

12. GSD3, dossier FIAF, manuscrit de 3 pages sans date, possiblement congrès de Rome, fin novembre 1949.

13. Éléments qui expliquent la cohésion de la profession, mobilisée lors de l'opposition aux accords Blum-Byrnes (1946-1948) avant d'obtenir du Centre national du cinéma une première loi d'aide à la production et à la diffusion (1953).

14. Dans l'entre-deux-guerres, on a déjà évoqué les réalisations de Ciné-Liberté, mais on pourrait également citer $\grave{A}$ propos de Nice (1930) de Jean Vigo, film indépendant, réalisé par un animateur de club.

15. Anonyme, «À nos lecteurs», Ciné-Club, nº 1, décembre 1949, p. 1.

\section{RÉFÉRENCES BIBLIOGRAPHIQUES}

Bloc-Delahaie 1950 : Pierre Bloc-Delahaie, «Échec au fisc, victoire du cinéma culturel ? ", L'Écran français, n' 266, 7 août 1950, p. 14.

Chevalier et Egly 1953: Jacques Chevalier et Max Egly (dir.), Regards neufs sur le cinéma, Paris, Seuil, 1953.

De Baecque 2003 : Antoine de Baecque, La cinéphilie: invention d'un regard, histoire d'une culture, 1944-1968, Paris, Fayard, 2003.

Gauthier 1999: Christophe Gauthier, La passion du cinéma. Cinéphiles, ciné-clubs et salles spécialisées à Paris de 1920 à 1929, Paris, AFRHC / École des Chartes, 1999.

Hamery 2008 : Roxane Hamery, Jean Painlevé, le cinéma au coeur de la vie, Rennes, Presses universitaires de Rennes, 2008.

Hamery 2012 : Roxane Hamery, "Les ciné-clubs dans la tourmente. La querelle du non-commercial (1948-1955)", Vingtième siècle, volume 115, n 3, 2012, p. 75-88.

Jeander 1949: Jeander, "Les ciné-clubs", dans Denis Marion (dir.), Le cinéma par ceux qui le font, Paris, Arthème-Fayard, 1949, p. 379-397.

Georges Sadoul et la Fédération française des ciné-clubs ou Contribution à une histoire des usages non commerciaux du cinéma 
Jullier et Leveratto 2010 : Laurent Jullier et Jean-Marc Leveratto, Cinéphiles et cinéphilies. Une histoire de la qualité cinématographique, Paris, Armand Colin, 2010.

Loyer 1992 : Emmanuelle Loyer, "Hollywood au pays des ciné-clubs (1947-1954)", Vingtième siècle, volume 33, n' 1, 1992, p. 45-55.

Pinel 1964: Vincent Pinel, Introduction au ciné-club. Histoire, théorie et pratique du ciné-club en France, Paris, Les éditions ouvrières, 1964.

Sadoul 1945: Georges Sadoul, "Les ciné-clubs et leurs histoires", Les Lettres françaises, $\mathrm{n}^{\circ} 72,8$ septembre 1945 , p. 5 .

Souillés-Debats 2017 : Léo Souillés-Debats, La culture cinématographique du mouvement ciné-club: une histoire de cinéphilie (1944-1999), Paris, AFRHC, 2017.

Vignaux 2011 : Valérie Vignaux, "Léon Moussinac et L'Humanité du cinéma. Cinéma militant et militantisme culturel dans l'entre-deux-guerres en France ", Études photographiques, $\mathrm{n}^{\circ} 27$, mai 2011, p. 200-239, http://etudesphotographiques.revues. org/3180.

Vignaux 2015: Valérie Vignaux, "Georges Sadoul aux Lettres françaises. Une critique de la contemporanéité", dans Laurent Jullier (dir.), Les films à voir cette semaine. Stratégies de la critique de cinéma, Paris, L'Harmattan, 2015, p. 55-76.

Vignaux 2016: Valérie Vignaux, "Georges Sadoul rédacteur en chef de Mon Camarade (1933-1939). Un magazine illustré pour une culture de jeunesse communiste?", Strene, n' 10, 2016, http://strenae.revues.org/1498.

Vignaux 2016a: Valérie Vignaux, "Georges Sadoul et le surréalisme ou Chronique d'un itinéraire intellectuel ", Annales Louis Aragon et Elsa Triolet, n 17, mars 2016, p. 15-45.

Zendel 1946: José Zendel, "Le rayonnement des ciné-clubs", L'Écran français, $\mathrm{n}^{\circ}$ 54, 10 juillet 1946, p. 14.

Zendel 1947: José Zendel, "Vingt-cinq ans de ciné-clubs (XIII). De la guerre à la Libération ", L'Écran français, n $105,1^{\text {er }}$ juillet 1947, p. 13.

\section{ABSTRACT}

\section{Georges Sadoul and the Fédération française des ciné-clubs, or Contribution to a History of Non- commercial Uses of Cinema Valérie Vignaux}

Drawing on the personal archives of Georges Sadoul, this article examines his reasons for joining the Fédération française des ciné-clubs in 1945 and for leaving it in 1952. Going against the grain of historical narratives, which see ciné-clubs as the agents of cinema's aesthetic recognition or status as heritage, the author sees them as contributing to a broader cultural movement, launched in the inter-war period by Léon Moussinac, for widespread cultural democratization and decentralization. This is a social understanding of an art form which gives nuance to the usual discourses around post-war cinephile activities. In addition, the article offers a new definition of popular education itself. 